linked mutation involved. Screening for CDKL5 mutations is recommended in children with epileptic encephalopathies of unknown origin and infantile spasms. (Moseley BD, Dhamija R, Wirrell EC, Nickel KC. Pediatr Neurol February 2012;46:101-105), (Response: Dr Moseley, Department of Neurology, Mayo Clinic, 200 First Street Southwest, Rochester, MN 55905. E-mail: moseley.brian@mayo.edu).

COMMENT. The authors list several genetic defects that predispose children to early-onset epileptic encephalopathies and infantile spasms, previously considered cryptogenic. The CDKL5 mutations are thought to influence brain development via a similar molecular pathway to $M E C P 2$, the mutation that causes the majority of Rett syndrome cases. However, none of the authors' $C D K L 5$ cases demonstrate the typical Rett syndrome-like phenotype with hand stereotypies, and only 1 has a characteristic microcephaly. Seizures with $C D K L 5$ mutations are refractory to treatment including the ketogenic diet, whereas the majority (56\%) of children with Rett syndrome and MECP2 mutations have treatment-responsive seizures. (Krajnc $N$ et al. J Child Neurol 2011;26:1429-1433). The authors' suggestion that acidosis is a possible mechanism of the ketogenic diet is contrary to earlier research conducted at the Mayo Clinic (Millichap JG et al. Amer J Dis Child 1962;107:593-604, and idem Epilepsia 1964;5:239-255).

KCNQ2 Encephalopathy, an emerging phenotype of a neonatal epileptic encephalopathy is reported in 8 patients with early onset intractable seizures (first week of life) with prominent tonic component. (Weckhuysen S, Mandelstam S, Suls A, et al. Ann Neurol January 2012;71:15-25). Seizures resolved by 3 years but residual intellectual disability and motor impairment were severe. EEG at onset showed a burstsuppression pattern or multifocal epileptiform activity. Early brain MRI showed hyperintensities in basal ganglia and thalamus that later resolved. KCNQ2 screening should be considered in the workup of refractory neonatal seizures of unknown origin.

\title{
HASHIMOTO ENCEPHALOPATHY AND STATUS EPILEPTICUS
}

A 12-year-old boy with Hashimoto encephalopathy and drug-resistant status epilepticus responsive to plasmapheresis is reported from Ankara University Medical School, Turkey. He was admitted with a right focal seizure, becoming secondary generalized tonic-clonic, refractory to treatment and necessitating a pentobarbital-induced coma. Recent history revealed a sudden change in personality, fever, headache, and fatigue, indicating limbic encephalitis. Serum anti-thyroid peroxidase antibody was elevated at $30 \mathrm{IU} / \mathrm{ml}$ (normal range, $0-9 \mathrm{IU} / \mathrm{ml}$ ). Treatment with iv immunoglobulin was ineffective, and plasmapheresis was performed, followed by levothyroxine and oral prednisolone $(2 \mathrm{mg} / \mathrm{kg} / \mathrm{day})$. The neurologic and psychiatric manifestations (orofacial dyskinesia, autonomic instability, emotional lability, and personality changes) decreased after the eighth plasmapheresis, and his examination was normal after 2 months. He was discharged taking prednisolone $(1 \mathrm{mg} / \mathrm{kg} /$ day $)$, levothyroxine, and antiepileptic drugs. (Bektas O, Yilmaz A, Kendirli T, Siklar Z, Deda G. Hashimoto encephalopathy causing drug-resistant status epilepticus treated with plasmapheresis. Pediatr Neurol February 2012;46:132-135).(Respond: Dr Bektas, Department of Pediatric Neurology, Ankara University Medical School, Ankara, Turkey. E-mail: bektasomer@gmail.com). 
COMMENT. Hashimoto encephalopathy (HE) is characterized by seizures, neurologic and psychiatric manifestations, and elevated titers of serum anti-thyroid antibodies. It is responsive to corticosteroids. Plasmapheresis is a novel method of acute treatment. HE should be considered, along with anti-N-methyl-D-aspartate-receptor, voltage-gated potassium channel antibody-associated limbic encephalitis, and herpes simplex virus encephalitis, in the differential diagnosis of a child with acute personality changes and seizures resistant to antiepileptic medication. The pathogenesis of HE is associated with high serum anti-thyroid antibody titers; thyroid hormone levels are usually normal or slightly low. An autoimmune disease process is likely.

\section{POLG NOVEL MUTATION WITH ALPERS SYNDROME}

Researchers at University Hospital, Berne, Switzerland describe the molecular genetic analysis of POLG in a 3.5 years old boy with VPA-induced fatal liver failure and encephalopathy (Alpers-Huttenlocher syndrome, AHS). Mutations in the POLG gene are a common cause of inherited mitochondrial disease in children and adults. They are involved with various neurodegenerative diseases, including Alpers syndrome, and result in accumulation of multiple mtDNA deletions and/or depletions of mtDNA in muscle, brain and liver. Some POLG mutations lead to a range of clinical phenotypes that predispose to fatal liver failure after exposure to VPA. POLG analysis in mitochondrial diseases helps in confirmation of AHS and optimizes clinical management. (Schaller A, Hahn D, Jackson CB, et al. Molecular and biochemical characterization of a novel mutation in POLG associated with Alpers syndrome. BMC Neurology 2011;11:4-11). (Respond: Dr Andre Schaller. E-mail: andre.schaller@insel.ch).

COMMENT. The study extends the list of $P O L G$ mutations associated with VPA hepatoxicity. A report of reversible valproate hepatotoxicity due to mutations in mitochondrial DNA polymerase gamma (POLG1) is cited. (McFarland R et al. Arch Dis Child 2008;93(2):151-153).

\section{DEMYELINATING DISEASE}

\section{HEAD AND BRAIN SIZE IN PEDIATRIC MULTIPLE SCLEROSIS}

Researchers at the Montreal Neurological Institute, the Hospital for Sick Children, Toronto, Canada; and Department of Neurology, Rennes, France conducted MRI measurements of whole brain and regional white matter, gray matter, and deep gray matter structure volumes in 38 patients (mean age 15.2+/-2.4 years) with pediatric-onset relapsing-remitting multiple sclerosis (MS). Mean age at MS onset was 12.1 years; mean disease duration 3.1 years. Values obtained from sex-matched healthy controls enrolled in the MRI Study of Normal Brain Development were used as controls. The intracranial volume and normalized brain volume $\mathrm{z}$ scores were significantly lower in patients with MS compared with controls. Thalamic volumes in MS patients were lower even after correction for global brain volume decreases. Reduced thalamic and brain volumes 